\title{
Evolution of Plasmodium vivax populations in border areas of the Greater Mekong Subregion during malaria elimination
}

\section{Yuling Li}

China Medical University

\section{Yubing Hu}

china medical university

\section{Yan Zhao}

china medical university

\section{Qinghui Wang} china medical university

\section{Huguette Gaelle Ngassa Mbenda}

university of south florida

\section{Veerayuth Kittichai} mahidol university

\section{Saranath Lawpoolsri} mahidol university

\section{Jetsumon Sattabongkot} madihol university

\section{Lynette Menezes}

university of south florida

Xiaoming Liu

university of south florida

Liwang Cui ( $\square$ liwangcui@usf.edu )

university of south florida

Yaming Cao ( $\square$ ymcao@cmu.edu.cn )

China Medical University https://orcid.org/0000-0003-3917-0958

\section{Research}

Keywords: Malaria, Plasmodium vivax, Epidemiology, Population genetics, Microsatellites, Greater Mekong Subregion

Posted Date: December 31st, 2019 
DOI: https://doi.org/10.21203/rs.2.19786/v1

License: (c) (1) This work is licensed under a Creative Commons Attribution 4.0 International License. Read Full License 


\section{Abstract}

\section{Background}

Countries within the Greater Mekong Subregion (GMS) of Southeast Asia have committed to eliminating malaria by 2030. Although malaria situation has greatly improved, Plasmodium vivax remains at international border regions. Therefore, to gain a better understanding of transmission dynamics, knowledge on the evolution of $P$. vivax populations after the scale-up of control interventions will guide more effective targeted control efforts.

\section{Methods}

We investigated genetic diversity and population structures in 206 longitudinally collected $P$. vivax clinical samples in two international border areas at the China-Myanmar border (CMB, n=50 in 2004 and n=52 in 2016) and western Thailand border ( $n=50$ in 2012 and $n=54$ in 2015). Parasites were genotyped using 10 microsatellite markers.

\section{Results}

Despite intensified control efforts, genetic diversity in the four populations remained high $\left(H_{E}=0.66-\right.$ 0.86). The proportions of polyclonal infections showed substantial decreases to 23.7 and $30.7 \%$ in the $\mathrm{CMB}$ and western Thailand, respectively, with corresponding decreases in the multiplicity of infection. Consistent with the shrinking map of malaria transmission in the GMS over time, there were also increases in multilocus linkage disequilibrium, suggesting of more fragmented and increasingly inbred parasite populations. There were considerable genetic differentiation and subdivision with the four tested populations. Various degrees of clustering were evident between the older parasite samples collected in 2004 at the CMB with the 2016 CMB and 2012 Thailand populations, suggesting some of these parasites had shared ancestry. In contrast, the 2015 Thailand population was genetically distinctive, which may reflect a process of population replacement. The moderately large effective population sizes and proportions of polyclonal infections highlight the necessity of further coordinated and integrated control efforts on both sides of the borders in the pursuit of malaria elimination.

\section{Conclusions}

With enhanced control efforts on malaria elimination, $P$. vivax population in the GMS has fragmented into a limited number of clustered foci, but the presence of large $P$. vivax reservoirs still sustains genetic diversity and transmission. These findings provide new insights into $P$. vivax transmission dynamics and population structure in this area.

\section{Background}

As the global incidence of malaria has been greatly reduced in recent years, Plasmodium vivax has become the main source of malaria infections in co-endemic areas [1]. This is also true for most areas of 
the Greater Mekong Subregion (GMS) in Southeast Asia [2], which is actively pursuing regional malaria elimination. This shift in species dominance in the face of intensified control efforts highlights the remarkable adaptive potential and relative resilience of $P$. vivax to control measures [3]. In recognizing the challenge for eliminating vivax malaria, the GMS countries planned to eliminate falciparum malaria by 2025 and all malaria by 2030 [4]. As the malaria elimination plan progresses in the GMS, malaria displayed increasing heterogeneity in distribution with transmission being concentrated along international borders [5]. These international borders are porous with intensive human migration, which poses a major threat of parasite introduction [6-8]. In addition, border regions have drastically different ecology, vector systems, human populations, and are subject to influences of wars and civil unrest; thus malaria transmission in border regions are often unstable [9]. While malaria surveillance has been strengthened in the GMS countries, it can be complemented by population genetic studies to define parasite diversity, population structure, and migration among geographically separated transmission hotspots [10].

Since a major mechanism of generating genetic diversity in the malaria parasites is meiotic recombination of parasite strains in mosquito vectors, genetic diversity is intrinsically linked to the transmission intensity [11]. Likewise, it is expected that reduction of parasite population in response to intensified interventions will lead to reduced parasite genetic diversity. However, $P$. vivax seems to defy this principle. In most of its geographic range, $P$. vivax showed high levels of genetic diversity as revealed by population genetic studies using various genotyping markers [12-21]. Paradoxically, even in low transmission settings, $P$. vivax still maintains high levels of diversity [22-26]. As a result, in areas of coendemicity, $P$. vivax and $P$. falciparum exhibit markedly different genetic diversity and population structures, with $P$. vivax showing more stable transmission patterns [14, 15, 27-29]. Newly introduced parasites in areas where malaria had been eliminated may initially display low-level genetic diversity or even clonality [30,31], but the parasites could rapidly reestablish diverse populations [32]. As a result, the shrinking $P$. vivax populations in areas with intensified control frequently showed relatively high genetic diversity, but substantial levels of multilocus linkage disequilibrium (LD) and population substructure [15, $17,23,33]$.

The scale-up of malaria control efforts in countries of the GMS has led to substantial changes in malaria epidemiology with a noticeable rising proportion of vivax malaria [5, 34]. In the China-Myanmar border (CMB) areas, P. vivax not only has become the predominant Plasmodium species in recent years [35], but also has caused several malaria outbreaks $[9,36]$. Even in the international border regions, malaria transmission is concentrated in separated hotspots, and among which gene flow is expected to be low. Final attacks to eliminate these hotspots, guided by accurate surveillance, will ensure the success of the regional elimination program. An improved understanding of the transmission dynamics and the adaptive responses of the parasites to the control measures will also be essential to guide and monitor the progress of the elimination campaign. Recent studies of $P$. vivax populations from international border regions of Thailand with microsatellite (MS) markers clearly detected parasite population division, consistent with the separation of these parasite populations by the malaria-free central region $[13,37]$. Here we employed longitudinally collected $P$. vivax clinical samples to determine the effect of intensified 
control efforts on genetic diversity and evolution of the parasite populations in two border regions of the GMS.

\section{Methods}

\section{Ethics statement}

Written informed consent was obtained from all $P$. vivax patients or their legal guardians for participants under the age of 18 years. This study was approved by the Institutional Review Boards of Department of Disease Control, Thailand Ministry of Health and the Pennsylvania State University. Use of the deidentified blood samples was approved by the Ethics Review Committee of China Medical University.

\section{Study sites and $P$. vivax clinical samples}

In order to investigate the evolution of the $P$. vivax population in response to intensified malaria control efforts in the GMS, P. vivax isolates were longitudinally collected from two different areas of the ChinaMyanmar and Thailand-Myanmar borders (S1 Fig). The CMB area has been traditionally malaria hyperendemic with both $P$. falciparum and $P$. vivax transmission. Although recent malaria control efforts have sharply reduced the $P$. falciparum incidence [35], $P$. vivax incidence remained consistently high and even experienced outbreaks in recent years $[9,36]$. In the CMB, 50 samples were collected in 2004 to represent the parasite population prior to the implementation of malaria elimination measures, while 52 samples collected in 2016 represented the parasites after scaling-up of malaria control. In Thailand, Tak province has been among the most malaria-endemic areas. In the past decade, malaria incidence in Tak has experienced more than 10-fold reduction, from 13,706 in 2012 to 1364 in 2016 . Fifty P. vivax clinical samples obtained in 2012 from Tak Province represented the parasites before the endorsement of malaria elimination plan, and were used to compare with samples collected from the same area in later years [13]. Finger-prick blood samples were collected from symptomatic patients attending local malaria clinics after obtaining informed consent. Malaria diagnosis was based on microscopy of Giemsa-stained thin and thick smears. For confirmed $P$. vivax cases, $100 \mu$ l of finger-prick blood were spotted onto Whatman filter paper, dried and stored in individually zipped plastic bags.

\section{Microsatellite genotyping}

Parasite genomic DNA was isolated from the dried blood spots on filter paper according to the protocol of QIAmp DNA Mini kit (Qiagen, Hilden, Germany). The final purified DNA was eluted into $35 \mathrm{ml}$ of elution buffer and used immediately or stored at $-20^{\circ} \mathrm{C}$ until further use.

A volume of $2 \mathrm{ml}$ of purified DNA was used as the template for malaria parasite detection by a genusspecific and species-specific nested PCR targeting the Plasmodium 18S rRNA genes [38]. Ten MS markers (MS1, MS2, MS5, MS6, MS7, MS9, MS10, MS12, MS15 and MS20) previously used to differentiate $P$. vivax populations in Thailand [13] were used for genotyping these $P$. vivax samples. A multiplex primary PCR was done using all 10 primer pairs and followed by singleplex secondary PCR with 
a fluorescently-labelled (6-FAM, VIC or NED) forward primer as previously described [39]. PCR products were used for GeneScan fragment analysis on an ABI3730xl capillary electrophoresis platform (Applied Biosystems) using the size standard LIZ500. Genotype calling was facilitated with GeneMapper Version 4.0. The predominant allele and any additional alleles with peak height at least one-third of the height of the predominant allele per locus were scored [40]. Genotyping success was defined as the presence of at least one allele at a given locus in a given sample.

\section{Data analysis}

Isolates containing more than one peak for any marker were considered to be multiple clone infections. The multiplicity of infection (MOI) was defined as the maximum number of alleles observed at any of the loci investigated. The mean $\mathrm{MOI}$ was calculated from the individual samples for each study site. Alleles were binned using the TANDEM software [41]. Isolates with one allele at all markers, were considered monoclonal infections. An infection was defined as polyclonal if more than one allele was observed at one or more loci. For isolates with more than one allele at any of the loci, the alleles with the highest peak were used to construct the dominant haplotypes as previously described [14]. Input files for the various population genetics software programs were created using CONVERT version 1.31.

\section{Population diversity and differentiation}

The indices of genetic diversity within populations, such as the number of polymorphic loci, the number of haplotypes $(\mathrm{Nh})$, the number of alleles $(\mathrm{Na})$, the mean allelic richness, and the expected heterozygosity $\left(H_{E}\right)$ were calculated using Arlequin version 3.11 [42] and GenAlEx version 6.5 [43]. In order to assess the

genetic differentiation among populations, pairwise comparisons were measured by calculating $F_{S T}$ using GenAIEx version 6.5. To estimate the partitioning of genetic variance for different hypothesized population groupings, we performed analysis of molecular variance (AMOVA) using GenAIEx version 6.5. The correlation between geographic and genetic distances was calculated by Mantel rank test in GenAlEx version 6.5.

\section{Linkage disequilibrium (LD)}

Multilocus LD in each population was calculated using the program LIAN 3.7 [44] with 50,000 iterations for burn-in and then 100,000 Markov Chain Monte Carlo (MCMC) iterations. Samples with missing data were excluded for this analysis. To avoid detecting false inbreeding resulting from clonal propagation and physical linkage, this analysis was performed for the combined dataset of single and dominant haplotypes and for unique haplotypes only. Since MS2 and MS5 both localize to chromosome 6 and MS12 and MS15 to chromosome 5, MS2 and MS12 were excluded as they had higher levels of missing data.

\section{Effective population size ( $\mathrm{Ne})$}

The effective population size was calculated using the stepwise mutation model (SMM) and infinite 
alleles model (IAM) as previously described [11]. Mutation rates for $P$. vivax are lacking and thus the $P$. falciparum mutation rate of $1.59 \times 10^{-4}\left(95 \%\right.$ confidence interval: $\left.6.98 \times 10^{-5}-3.7 \times 10^{-4}\right)$ was used [45].

\section{Bottleneck analysis}

A heterozygosity excess test at the population level was used to detect the recent population bottleneck using BOTTLENECK 1.2.02 [46] with the SMM [47]. The Wilcoxon signed rank test, a robust statistic for testing less than 20 polymorphic loci, was executed in the model in order to ascertain the probability of significant heterozygote excess. Since the method implemented in the bottleneck has low power [48] unless the decline is greater than $90 \%$, the Garza-Williamson index was computed using Arlequin version 3.11. The Garza-Williamson index is the mean ratio of the number of alleles at a given locus to the range in allele size, i.e., $M=(k / r)$, where $k$ is the number of alleles and $r$ is the allelic range (i.e., the difference in repeat units between the shortest and the longest alleles at a locus) [49]. This measure is based on the assumption that in a bottleneck event, the number of alleles decreases faster than the allelic range because the latter is only reduced if the shortest and/or longest allele is lost, whereas the loss of any allele reduces the former. For the Garza-Williamson index, $M<0.68$ indicates a bottleneck, whereas $M>$ 0.80 indicates no reduction of effective population size.

\section{Population structure}

Deviations from Hardy-Weinberg equilibrium could indicate the presence of population structure or inbreeding [50]. To investigate whether haplotypes cluster into distinct genetic populations (K) among the defined geographic areas, we conducted a Bayesian analysis of population structure using STRUCTURE version 2.3.2 [51]. The admixture model was used and the posterior probability of the grouping number ( $\mathrm{K}$ = 1 10) was estimated by the MCMC method with 10 separate runs to evaluate the consistency of the results. Each run was estimated as 10,000 steps with 100,000-step burn-in. The best-fit number of grouping was evaluated using $\triangle K$ in the STRUCTURE HARVESTER version 0.6 .93 tool $[52,53]$. The CLUMPP version 1.1.2 [54] and DISTRUCT 1.1(Rosenberg NA, 2004) were used to display the partitioning clusters.

To visualize genetic relationships among the parasite isolates from the four populations, an individualbased principal coordinate analysis (PCOA) was conducted in GenAlEx version 6.5 using the genetic distances among MS genotypes. In addition, phylogenetic relationships amongst $P$. vivax isolates were analyzed using the Neighbor-Joining method [55] implemented in MEGA7 [56]. Minimum spanning tree of parasite genotypes constructed by the goeBURST algorithm using the Phyloviz software v1.1 (http://www.phyloviz.net/)

\section{Results}

\section{Within-host and Population Diversity}


A total of $206 P$. vivax isolates from two areas of the GMS were genotyped at 10 MS markers. Complete genotyping data for all 10 loci were obtained for 166 (80.5\%) isolates. Samples with fewer than five MS markers genotyped were removed from analysis. The predominant allele of each locus in each sample was used for population genetic analysis. The $10 \mathrm{MS}$ markers all were highly polymorphic with each one having 2 - 20 alleles when all parasite populations were considered (S1 Table). Allele frequencies varied among the four populations. In the CMB samples, MS2 was the most diverse in 2004 with 20 alleles, whereas MS7 was the most diverse in 2016 with 16 alleles, followed by MS9 with 15 alleles. In the 2012 samples in western Thailand, MS20 was the most diverse with 18 alleles, followed by MS2 with 16 alleles. The 2015 samples from three provinces of Thailand also had MS2 as the most diverse marker [13]. Therefore, MS2 appeared to be among the most diverse markers in malaria-endemic areas in the GMS. In comparison, the least diverse MS markers differed among the populations: MS6 in the 2004 CMB population with 6 alleles, MS20 in the 2016 CMB population with two alleles; MS6 and MS12 equally least diverse in 2012 western Thailand with 7 alleles; and MS1 in 2015 western Thailand with 6 alleles (S1 Table).

Overall, 72 (34.9\%) parasite isolates contained polyclonal infections (more than one peak for at least one MS marker), and the proportions of multiclonal infections were significantly different among the four parasite populations ( $P<0.05$, Pearson Chi-square test) (Table 1). At both sites, along with the reduction in malaria incidence, there were substantial decreases in the proportion of multiclonal infections $(P<$ 0.05 , Pearson Chi-square test). At the CMB, compared with the $48.0 \%$ polyclonal infections observed in 2004, this proportion decreased to $30.7 \%$ in 2016 . Similarly, in western Thailand, the percentage of polyclonal infections also experienced a considerable decrease from $40.0 \%$ in 2012 to $23.7 \%$ in 2016 . Accordingly, this was reflected in the reduction of mean MOI from 1.48 in 2004 to 1.33 in 2016 in CMB, and from 1.50 in 2012 to 1.36 in 2015 in western Thailand (Table 1). The genetic diversity was correspondingly reflected in allele richness in these parasite populations (Table 1). At the CMB, comparison of allele richness in parasite populations in 2004 and 2016 showed a substantial reduction from 12.2 to 8.8 . In contrast, the allele richness in parasite populations in western Thailand did not show much changes between 2012 and 2015.

Despite the overall reduction in $\mathrm{MOI}$, the genetic diversity of parasite populations at both sites remained high (Table 1). Haplotype diversity was high; a total of 162 haplotypes were observed and no haplotypes were shared among the four populations. The 2015 Thailand parasite population harbored the greatest number of haplotypes (Table 1). A slight decrease in the expected heterozygosity was observed at the CMB from 0.76 in 2004 to 0.66 in 2016, although this did not reach statistical significance $(P>0.05)$. In comparison, the genetic diversity of parasite population in western Thailand had a slight increase from 2012 to 2015 (Table 1). This high genetic diversity may reflect substantially large parasite populations sustaining continued transmission at these two border areas. With both the SMM and IAM models, the effective population size $N_{e}$ at the CMB was moderate and showed a $\sim 2$-fold decrease over the past decade, whereas $N_{e}$ in western Thailand remained high and even had a $\sim 2$-fold increase in recent years

(Table 2). 


\section{Multilocus LD and Population Bottlenecks}

Though haplotype sharing among the four parasite populations was not detected, the $2004 \mathrm{CMB}$ and 2015 Thailand populations had low degrees of haplotype sharing within the populations. In the 2004 CMB population, two parasite isolates carried the same haplotypes 13 and 22, respectively (S2 Table). In the 2015 Thailand parasite population, identical haplotypes 25, 79, 92, and 109 were shared by two, two, three, and four parasites, respectively [13]. The standardized index of association $I^{S} \mathrm{~A}$ used to measure the degree of inbreeding revealed significant multilocus LD in the $P$. vivax populations from the both CMB ( $P$ $<0.0001)$ and western Thailand $(P<0.0001)$ (Table 3). Multilocus LD was also retained when only unique haplotypes or only one MS marker per chromosome (excluding MS2 and MS12) was used, suggesting the presence of significant inbreeding in all these populations. Considering temporal changes in multilocus $L D$, there was an increasing trend of $L D$ in parasite populations at the $C M B$ from $2004\left(I^{S} A=\right.$ $0.0236)$ to $2016\left(I^{S} \mathrm{~A}=0.0329\right)$ and in Tak Province of Thailand from $2012\left(I^{\mathrm{S}} \mathrm{A}=0.0366\right)$ to $2015\left(I^{\mathrm{S}} \mathrm{A}=\right.$ 0.0586) (Table 3), which may contribute to the potential effect of population reduction in both sites with the scale-up of malaria control measures.

To detect whether there were significant changes in the parasite population size, we performed BOTTLENECK analysis under SMM and computed the Garza-Williamson index statistic (Table 4). The model detected significant deficiency in $\mathrm{H}_{\mathrm{E}}$ from the mutation-drift equilibrium, indicating events of population size reduction with possible clonal expansion in all four populations. Likewise, the mean G-W index was less than 0.68 in all the four populations, also suggesting a reduction of the effective population size.

\section{Genetic Differentiation and Population Structure}

Genetic differentiation of the $P$. vivax populations in the GMS were evaluated by estimating the Wright's fixation index $F_{\mathrm{ST}}$. As expected, the contemporary $P$. vivax populations from the $\mathrm{CMB}$ and western Thailand showed considerable genetic differentiation $\left(F_{\mathrm{ST}}=0.169\right.$ and 0.237$)$. The CMB $P$. vivax populations showed moderate differentiation between 2004 and $2016\left(F_{\mathrm{ST}}=0.081\right)$, whereas the western Thailand parasite populations had substantial differentiation between 2012 and $2015\left(F_{\text {ST }}=0.133\right.$, Table 5). Interestingly, the $2004 \mathrm{CMB}$ parasite population also showed little genetic differentiation from those collected from the 2012 Thailand population $\left(F_{S T}=0.064\right)$, but significant genetic differentiation from the 2015 Thailand population $\left(F_{S T}=0.172\right)$. The Mantel test on correlation between pairwise genetic and geographical distances indicated a weak, insignificant positive association between genetic and geographical distances (Mantel rank test, $r^{2}=0.0297, p=0.44$ ). Analysis of molecular variation (AMOVA) indicated $87 \%$ of the variation was attributed to variations within populations, and $13 \%$ among populations.

STRUCTURE analysis showed a clear distribution pattern of parasite haplotypes and demonstrated multiple sub-populations (Fig 1A). Most parasites from Thailand collected in 2015 were separated from all other populations. Using the delta $\mathrm{K}$ method, the most likely number of sub-populations was identified 
as 2 (S2 Fig). At $\mathrm{K}=3$, populations collected in Thailand in 2015 formed a separate cluster, as well as the CMB samples collected in 2016, with admixture in samples from the CMB in 2004 and Thailand 2012. At $\mathrm{K}=4$, admixing between the CMB 2004 and Thailand 2012 parasite populations became more evident (Fig 1A), which corroborated the weak genetic differentiation between these two populations from $F_{\mathrm{ST}}$ analysis $\left(F_{\mathrm{ST}}=0.064\right.$, Table 5). At $\mathrm{K}=5$, parasites in 2004 from the $\mathrm{CMB}$ and 2012 from Thailand were substantially admixed (Fig 1A), suggesting similar ancestry for parasites from the CMB and 2012 Thailand.

In line with the STRUCTURE analysis, PCA confirmed the genetic separation of the four parasite populations. PC1 and PC2 explained $7.37 \%$ and $5.16 \%$ of the variances, respectively, and grouped the parasites into two main clusters. The three more contemporary samples Thailand 2015, Thailand 2012 and CMB 2016 formed individual clusters with no overlap, whereas the older parasite population CMB 2004 showed haplotype admixture with Thailand 2012 and CMB 2016 (Fig 1B). Such a finding was further illustrated with phylogenetic and network analyses.

The phylogenetic tree also supports the finding of substantial regional population structure and local clustering of haplotypes (Fig 2A). Clades 1 and 2 contained parasites predominantly from the CMB 2004 and 2016, which more or less reflected the lower right coordinate of the PCA analysis to show extensive clustering of parasites collected most recently from Myanmar with earlier parasite samples from the rest of the GMS (Fig 1B). Clade 3 and 4 parasites were predominantly from Thailand collected in 2012 and 2015, respectively. Similarly, network analysis roughly divided parasites into four clusters, with each cluster being dominated by parasites from a single population (Fig 2B). Cluster 4 contained mostly parasites from western Thailand 2015. Cluster 3 contained mostly parasites from western Thailand collected in 2012, while it also contained a few haplotypes from CMB border in 2004. Clusters 1 and 2 were dominated by haplotypes from CMB 2016 and 2004, respectively. Parasites collected most recently in 2016 from CMB were mainly found in clusters 1 and 2, where they showed close relationships with haplotypes from the earlier parasite population collected in 2004.

\section{Discussion}

The emergence of artemisinin resistance in P. falciparum has escalated the urgency to eliminate this parasite in in the GMS to prevent a catastrophic spread of artemisinin-resistant parasites to Africa. With the overall scale-up of control efforts including increased coverage of endemic population with longlasting insecticide treated net distribution, effective management of clinical cases with sensitive diagnosis and treatment, and heightened surveillance such as implementation of the 1-3-7 strategy, malaria incidence in the GMS has continually declined [57]. As found in other malaria-endemic regions with sympatric transmission of P. falciparum and P. vivax, P. vivax is less affected by conventional control measures due to its unique biology such as formation of hypnozoites enabling relapses and early gametocytogenesis to enable transmission before treatment is initiated. With P. vivax becoming dominant in most parts of the GMS, a better understanding of transmission dynamics and population structure of this parasite is crucial to guide targeted control efforts and to achieve the goal of malaria 
elimination under the changing settings of malaria epidemiology [58]. Thus, this study aimed to study the evolution of parasite population in the GMS during the elimination process with samples longitudinally collected in two international border areas before and after the scale-up of malaria control interventions. Our study demonstrated that despite intensified control efforts in the GMS, there were only moderate levels of reduction in parasite genetic diversity and the proportion of polyclonal infections. Meanwhile the $P$. vivax parasite displayed fragmented population structure with substantial genetic differentiation. We discuss the possible epidemiological processes driving these patterns of diversity and population structure.

The GMS is malaria hypoendemic with malaria transmission occurring along international borders. In both border regions, there are camps for refugees or internally displayed people (IDP) established, where there is constant human population movement. Genetic diversity of $P$. vivax populations in the two border regions of the GMS was moderately high with the expected heterozygosity ranging from 0.66 to 0.86 . Compared to previous studies which used a nearly identical set of microsatellite markers, the $H_{E}$ values in our study were remarkably close to those from low-to-moderate transmission areas in Asia such as Vietnam (0.68), Thailand (0.76), central China (0.816), Laos (0.75) and India (0.72), as well as South Americas such as Colombia (0.79) and Brazil (0.71) [22, 29, 30, 59, 60]. Despite strengthened malaria control efforts in both regions, $\mathrm{H}_{\mathrm{E}}$ at the $\mathrm{CMB}$ only decreased slightly over a 10-year period and remained relatively unchanged in western Thailand during the past few years. This is consistent with our earlier finding of stable $H_{E}$ in parasite populations in the CMB during the years 2011-2013. The high genetic diversity in low transmission areas is a common phenomenon that has been reported in numerous $P$. vivax-endemic areas [22-24, 28]. Reason for this is not completely clear but is considered to be multifactorial [61]. Of direct relevance to the border settings is the mobility of the camp and border populations along the borders, which would introduce new parasites and enhance genetic diversity of the parasite populations. Our earlier study provided evidence on the existence of extensive gene flow among border communities and across international borders [16]. Unlike international borders are present as a potential barrier for the spread of $P$. falciparum strains in the GMS [62], landscape factors at the CMB do not appear to impede gene flow [16]. Cross-border frequent human migration facilitates the importation of malaria into low-transmission areas and this represents a major challenge to malaria elimination in the GMS [63].

Unlike the relatively stable genetic diversity over time, both border regions showed substantial reduction (17-18\%) in the proportion of polyclonal infections and MOI. However, at the CMB, the proportion of polyclonal infections was unchanged compared to that determined in clinical $P$. vivax samples collected earlier. Nevertheless, compared to the hypoendemic settings in central China and Malaysia and hyperendemic settings such as Papua New Guinea, Cambodia, Indonesia and Ethiopia [15, 22, 64-67], the proportions of polyclonal infections in parasite samples from recent years remained moderately high $(23 \%$ and $30 \%$ ) in these two border regions of the GMS. In addition, parasite populations in these regions remained relatively large, especially at the western Thailand border. Multiclonal infection, together with the large population size, will increase the chance of recombination, resulting in the observed high genetic 
diversity. It is therefore important to further enhance local malaria control efforts to achieve substantial reduction of the parasite populations in these transmission hotspots.

Another noticeable change is the detection of significant multilocus LD in the parasite populations despite high genetic diversity. This again seems to be a common finding in many vivx low-endemicity settings such as Indonesia, Papua New Guinea, South Korea, India, Vietnam, Colombia, and Brazil [15, 59, $61][32,68,69]$. Significant $L D$ against a background of high diversity may reflect the existence of multiple spatially clustered infections within a defined population, which might have arisen from rapid reduction in transmission and effective population size as malaria control interventions have intensified in the GMS. The overall trend of increasing LD in both sites and elsewhere in Thailand [13] is a demonstration of reduced overall parasite genetic complexity during malaria elimination.

Across different regions of Thailand, we have detected substantial parasite population differentiation [13]. This study also detected considerable divergence of the parasite populations across both time and space. There were no haplotype sharing among the four populations, and most of the parasites from individual populations fell into distinct clades or clusters from phylogenetic and network analyses, demonstrating that the parasite populations in the GMS have become fragmented with increasingly inbred. International border regions of the GMS showed high malaria transmission areas that were largely connected a decade ago [70]. Consistently, the older parasite population from the CMB 2004 showed remarkable degrees of clustering with the CMB 2016 and Thailand 2012 samples, which may reflect shared ancestry of these parasites in the recent past. In contrast, the current malaria map illustrated the presence of separated pockets of transmission foci from intensified control efforts of the malaria elimination campaign, which limit gene flow within GMS. The genetic differentiation between the contemporary parasite populations is an indication of independent evolution of these isolated parasite populations as also documented for the $P$. falciparum parasites [71]. In addition, heterogeneity within vector populations could affect transmission [72] and population structure of the parasites [73]. The notable shift in vector populations observed in western Thailand may also be responsible for shaping the parasite population structure [74]. Moreover, the parasite populations studied here have undergone apparent population bottlenecks, suggesting of clonal expansion of parasite isolates, or limited vectors or human movement within the defined geographic areas. Relaxation of control efforts could lead to clonal expansion of the residual or introduced parasites, as has been identified in southern Thailand [31, 75]. At the $\mathrm{CMB}$, the epidemic malaria transmission documented in the IDP camps and surrounding villages in 2013 and the detected reduction of effective population size may result from expansion of certain parasite isolates such as those that are resistant to the frontline treatment $[76,77]$. Interestingly, the Thailand 2015 samples were quite genetically distinct, and even differed considerably from the 2012 parasite from the same region, which may suggest population replacement. This is plausible as the border parasite populations are subject to extensive parasite introduction [6], and introduced parasites are capable of re-establishing a more diverse population within a short period [32].

\section{Declarations}




\section{Funding}

This study was supported by grants from the National Institute of Allergy and Infectious Diseases (U19 Al089672), National Institutes of Health, USA.

\section{Authors' contributions}

YL, YH, LC, and YC conceived and designed the study; YL, YH, YZ, VK, SL and JS

collected the samples and data; $\mathrm{YL}, \mathrm{YH}, \mathrm{YZ}, \mathrm{QW}, \mathrm{HGNM}$ performed analyses and drafted the manuscript; $\mathrm{LM}, \mathrm{XL}, \mathrm{LC}$ and $\mathrm{YC}$ participated in data analysis and interpreted the results. All authors read and approved the final manuscript.

\section{Availability of data and materials}

The datasets supporting the conclusions of this article are available in Additional files.

\section{Ethics approval}

Ethical approvals were obtained from the institutional review boards of China Medical University, Thai Ministry of Health and Pennsylvania State University.

\section{Consent for publication}

Not applicable.

\section{Competing interests}

The authors declare that they have no competing interests.

\section{References}

1. Cotter C, Sturrock HJ, Hsiang MS, Liu J, Phillips AA, Hwang J, Gueye CS, Fullman N, Gosling RD, Feachem RG: The changing epidemiology of malaria elimination: new strategies for new challenges. Lancet 2013, 382:900-911.

2. WHO: World Malaria Report 2018. 2018.

3. Howes RE, Battle KE, Mendis KN, Smith DL, Cibulskis RE, Baird JK, Hay SI: Global Epidemiology of Plasmodium vivax. Am J Trop Med Hyg 2016, 95:15-34.

4. WHO: Eliminating malaria in the Greater Mekong Subregion: United to end a deadly disease. https://wwwwhoint/malaria/publications/atoz/eliminating-malaria-greater-mekong/en/ 2016.

5. Cui L, Cao Y, Kaewkungwal J, Khamsiriwatchara A, Lawpoolsri S, Soe TN, Kyaw MK, Sattabongkot J: Malaria Elimination in the Greater Mekong Subregion: Challenges and Prospects. In: Towards Malaria Elimination: A Leap Forward. Edited by Manguin S, Dev V: IntechOpen; 2018: 179-200. 
6. Parker DM, Carrara VI, Pukrittayakamee S, McGready R, Nosten FH: Malaria ecology along the Thailand-Myanmar border. Malar J 2015, 14:388.

7. Sriwichai P, Karl S, Samung Y, Kiattibutr K, Sirichaisinthop J, Mueller I, Cui L, Sattabongkot J: Imported Plasmodium falciparum and locally transmitted Plasmodium vivax: cross-border malaria transmission scenario in northwestern Thailand. Malar J 2017, 16:258.

8. Zhou G, Sun L, Xia R, Duan Y, Xu J, Yang H, Wang Y, Lee MC, Xiang Z, Yan G et al: Clinical malaria along the China-Myanmar border, Yunnan Province, China, January 2011-August 2012. Emerg Infect Dis 2014, 20:675-678.

9. Zhou G, Lo E, Zhong D, Wang X, Wang Y, Malla S, Lee MC, Yang Z, Cui L, Yan G: Impact of interventions on malaria in internally displaced persons along the China-Myanmar border: 20112014. Malar J 2016, 15:471.

10. Arnott A, Barry AE, Reeder JC: Understanding the population genetics of Plasmodium vivax is essential for malaria control and elimination. Malar J 2012, 11:14.

11. Anderson TJ, Haubold B, Williams JT, Estrada-Franco JG, Richardson L, Mollinedo R, Bockarie M, Mokili J, Mharakurwa S, French $\mathrm{N}$ et al: Microsatellite markers reveal a spectrum of population structures in the malaria parasite Plasmodium falciparum. Mol Biol Evol 2000, 17:1467-1482.

12. Koepfli C, Rodrigues PT, Antao T, Orjuela-Sanchez P, Van den Eede P, Gamboa D, van Hong N, Bendezu J, Erhart A, Barnadas C et al: Plasmodium vivax Diversity and Population Structure across Four Continents. PLoS Negl Trop Dis 2015, 9:e0003872.

13. Kittichai V, Koepfli C, Nguitragool W, Sattabongkot J, Cui L: Substantial population structure of Plasmodium vivax in Thailand facilitates identification of the sources of residual transmission. PLoS Negl Trop Dis 2017, 11:e0005930.

14. Jennison C, Arnott A, Tessier N, Tavul L, Koepfli C, Felger I, Siba PM, Reeder JC, Bahlo M, Mueller I et al: Plasmodium vivax populations are more genetically diverse and less structured than sympatric Plasmodium falciparum populations. PLoS Negl Trop Dis 2015, 9:e0003634.

15. Noviyanti R, Coutrier F, Utami RA, Trimarsanto H, Tirta YK, Trianty L, Kusuma A, Sutanto I, Kosasih A, Kusriastuti $R$ et al: Contrasting Transmission Dynamics of Co-endemic Plasmodium vivax and $P$. falciparum: Implications for Malaria Control and Elimination. PLoS Negl Trop Dis 2015, 9:e0003739.

16. Lo E, Lam N, Hemming-Schroeder E, Nguyen J, Zhou G, Lee MC, Yang Z, Cui L, Yan G: Frequent Spread of Plasmodium vivax Malaria Maintains High Genetic Diversity at the Myanmar-China Border without Distance and Landscape Barriers. J Infect Dis 2017.

17. Waltmann A, Koepfli C, Tessier N, Karl S, Fola A, Darcy AW, Wini L, Harrison GLA, Barnadas C, Jennison $\mathrm{C}$ et al: Increasingly inbred and fragmented populations of Plasmodium vivax associated with the eastward decline in malaria transmission across the Southwest Pacific. PLoS Negl Trop Dis 2018, 12:e0006146.

18. Rodrigues PT, Alves JM, Santamaria AM, Calzada JE, Xayavong M, Parise M, da Silva AJ, Ferreira $\mathrm{MU}$ : Using mitochondrial genome sequences to track the origin of imported Plasmodium vivax infections diagnosed in the United States. Am J Trop Med Hyg 2014, 90:1102-1108. 
19. Hupalo DN, Luo Z, Melnikov A, Sutton PL, Rogov P, Escalante A, Vallejo AF, Herrera S, Arevalo-Herrera M, Fan Q et al: Population genomics studies identify signatures of global dispersal and drug resistance in Plasmodium vivax. Nat Genet 2016, 48:953-958.

20. Pearson RD, Amato R, Auburn S, Miotto O, Almagro-Garcia J, Amaratunga C, Suon S, Mao S, Noviyanti R, Trimarsanto $\mathrm{H}$ et al: Genomic analysis of local variation and recent evolution in Plasmodium vivax. Nat Genet 2016, 48:959-964.

21. Baniecki ML, Faust AL, Schaffner SF, Park DJ, Galinsky K, Daniels RF, Hamilton E, Ferreira MU, Karunaweera ND, Serre D et al: Development of a single nucleotide polymorphism barcode to genotype Plasmodium vivax infections. PLoS Negl Trop Dis 2015, 9:e0003539.

22. Liu Y, Auburn S, Cao J, Trimarsanto H, Zhou H, Gray KA, Clark TG, Price RN, Cheng Q, Huang R et al: Genetic diversity and population structure of Plasmodium vivax in Central China. Malar J 2014, $13: 262$.

23. Gunawardena S, Ferreira MU, Kapilananda GM, Wirth DF, Karunaweera ND: The Sri Lankan paradox: high genetic diversity in Plasmodium vivax populations despite decreasing levels of malaria transmission. Parasitology 2014, 141:880-890.

24. Friedrich LR, Popovici J, Kim S, Dysoley L, Zimmerman PA, Menard D, Serre D: Complexity of Infection and Genetic Diversity in Cambodian Plasmodium vivax. PLoS Negl Trop Dis 2016, 10:e0004526.

25. Zhong D, Lo E, Wang X, Yewhalaw D, Zhou G, Atieli HE, Githeko A, Hemming-Schroeder E, Lee MC, Afrane $Y$ et al: Multiplicity and molecular epidemiology of Plasmodium vivax and Plasmodium falciparum infections in East Africa. Malar J 2018, 17:185.

26. Pacheco MA, Schneider KA, Cespedes N, Herrera S, Arevalo-Herrera M, Escalante AA: Limited differentiation among Plasmodium vivax populations from the northwest and to the south Pacific Coast of Colombia: A malaria corridor? PLoS Negl Trop Dis 2019, 13:e0007310.

27. Gray KA, Dowd S, Bain L, Bobogare A, Wini L, Shanks GD, Cheng Q: Population genetics of Plasmodium falciparum and Plasmodium vivax and asymptomatic malaria in Temotu Province, Solomon Islands. Malar J 2013, 12:429.

28. Fola AA, Harrison GLA, Hazairin MH, Barnadas C, Hetzel MW, Iga J, Siba PM, Mueller I, Barry AE: Higher Complexity of Infection and Genetic Diversity of Plasmodium vivax Than Plasmodium falciparum Across All Malaria Transmission Zones of Papua New Guinea. Am J Trop Med Hyg 2017, 96:630-641.

29. Ferreira MU, Karunaweera ND, da Silva-Nunes M, da Silva NS, Wirth DF, Hartl DL: Population structure and transmission dynamics of Plasmodium vivax in rural Amazonia. J Infect Dis 2007, 195:12181226.

30. Iwagami M, Fukumoto M, Hwang SY, Kim SH, Kho WG, Kano S: Population structure and transmission dynamics of Plasmodium vivax in the Republic of Korea based on microsatellite DNA analysis. PLoS Negl Trop Dis 2012, 6:e1592. 
31. Putaporntip C, Miao J, Kuamsab N, Sattabongkot J, Sirichaisinthop J, Jongwutiwes S, Cui L: The Plasmodium vivax merozoite surface protein 3 beta sequence reveals contrasting parasite populations in southern and northwestern Thailand. PLoS Negl Trop Dis 2014, 8:e3336.

32. Kim JY, Goo YK, Zo YG, Ji SY, Trimarsanto H, To S, Clark TG, Price RN, Auburn S: Further Evidence of Increasing Diversity of Plasmodium vivax in the Republic of Korea in Recent Years. PLoS One 2016, 11:e0151514.

33. Delgado-Ratto C, Gamboa D, Soto-Calle VE, Van den Eede P, Torres E, Sanchez-Martinez L, ContrerasMancilla J, Rosanas-Urgell A, Rodriguez Ferrucci H, Llanos-Cuentas A et al: Population Genetics of Plasmodium vivax in the Peruvian Amazon. PLoS Negl Trop Dis 2016, 10:e0004376.

34. Baird JK: Asia-Pacific malaria is singular, pervasive, diverse and invisible. Int J Parasitol 2016.

35. Li N, Parker DM, Yang Z, Fan Q, Zhou G, Ai G, Duan J, Lee MC, Yan G, Matthews SA et al: Risk factors associated with slide positivity among febrile patients in a conflict zone of north-eastern Myanmar along the China-Myanmar border. Malar J 2013, 12:361.

36. Geng J, Malla P, Zhang J, Xu S, Li C, Zhao Y, Wang Q, Kyaw MP, Cao Y, Yang Z et al: Increasing trends of malaria in a border area of the Greater Mekong Subregion. Malar J 2019, 18:309.

37. Congpuong K, Ubalee R: Population Genetics of Plasmodium vivax in Four High Malaria Endemic Areas in Thailand. Korean J Parasitol 2017, 55:465-472.

38. Wampfler R, Mwingira F, Javati S, Robinson L, Betuela I, Siba P, Beck HP, Mueller I, Felger I: Strategies for detection of Plasmodium species gametocytes. PLoS One 2013, 8:e76316.

39. Koepfli C, Timinao L, Antao T, Barry AE, Siba P, Mueller I, Felger I: A Large Plasmodium vivax Reservoir and Little Population Structure in the South Pacific. PLoS One 2013, 8:e66041.

40. Anderson TJ, Su XZ, Bockarie M, Lagog M, Day KP: Twelve microsatellite markers for characterization of Plasmodium falciparum from finger-prick blood samples. Parasitology 1999, 119 (Pt 2):113-125.

41. Matschiner M, Salzburger W: TANDEM: integrating automated allele binning into genetics and genomics workflows. Bioinformatics 2009, 25:1982-1983.

42. Excoffier L, Laval G, Schneider S: Arlequin (version 3.0): an integrated software package for population genetics data analysis. Evol Bioinform Online 2007, 1:47-50.

43. Peakall R, Smouse PE: GenAIEx 6.5: genetic analysis in Excel. Population genetic software for teaching and research-an update. Bioinformatics 2012, 28:2537-2539.

44. Haubold B, Hudson RR: LIAN 3.0: detecting linkage disequilibrium in multilocus data. Linkage Analysis. Bioinformatics 2000, 16:847-848.

45. Anderson TJ, Su XZ, Roddam A, Day KP: Complex mutations in a high proportion of microsatellite loci from the protozoan parasite Plasmodium falciparum. Mol Ecol 2000, 9:1599-1608.

46. Cornuet JM, Luikart G: Description and power analysis of two tests for detecting recent population bottlenecks from allele frequency data. Genetics 1996, 144:2001-2014. 
47. Shriver MD, Jin L, Chakraborty R, Boerwinkle E: VNTR allele frequency distributions under the stepwise mutation model: a computer simulation approach. Genetics 1993, 134:983-993.

48. Williamson-Natesan EG: Comparison of methods for detecting bottlenecks from microsatellite loci. Conservation Genetics 2005, 6:551-562.

49. Garza JC, Williamson EG: Detection of reduction in population size using data from microsatellite loci. Mol Ecol 2001, 10:305-318.

50. Hartl DL, Clark GC: Principles of Population Genetics. . Sinauer Associates, Sunderland 1997.

51. Pritchard JK, Stephens M, Donnelly P: Inference of population structure using multilocus genotype data. Genetics 2000, 155:945-959.

52. Earl DA, VonHoldt BM: STRUCTURE HARVESTER: a website and program for visualizing STRUCTURE output and implementing the Evanno method. Conservation Genet Resour 2012, 4:359-361.

53. Evanno G, Regnaut S, Goudet J: Detecting the number of clusters of individuals using the software STRUCTURE: a simulation study. Mol Ecol 2005, 14:2611-2620.

54. Jakobsson M, Rosenberg NA: CLUMPP: a cluster matching and permutation program for dealing with label switching and multimodality in analysis of population structure. Bioinformatics 2007, 23:1801-1806.

55. Saitou N, Nei M: The neighbor-joining method: a new method for reconstructing phylogenetic trees. Mol Biol Evol 1987, 4:406-425.

56. Kumar S, Stecher G, Tamura K: MEGA7: Molecular Evolutionary Genetics Analysis Version 7.0 for Bigger Datasets. Mol Biol Evol 2016, 33:1870-1874.

57. WHO: World Malaria Report. https://wwwwhoint/malaria/publications/world-malaria-report2018/en/ 2018.

58. Barry AE, Waltmann A, Koepfli C, Barnadas C, Mueller I: Uncovering the transmission dynamics of Plasmodium vivax using population genetics. Pathog Glob Health 2015, 109:142-152.

59. Hong NV, Delgado-Ratto C, Thanh PV, Van den Eede P, Guetens P, Binh NT, Phuc BQ, Duong TT, Van Geertruyden JP, D'Alessandro U et al: Population Genetics of Plasmodium vivax in Four Rural Communities in Central Vietnam. PLoS Negl Trop Dis 2016, 10:e0004434.

60. Imwong M, Nair S, Pukrittayakamee S, Sudimack D, Williams JT, Mayxay M, Newton PN, Kim JR, Nandy A, Osorio L et al: Contrasting genetic structure in Plasmodium vivax populations from Asia and South America. Int J Parasitol 2007, 37:1013-1022.

61. Fola AA, Nate E, Abby Harrison GL, Barnadas C, Hetzel MW, Iga J, Siba P, Mueller I, Barry AE: Nationwide genetic surveillance of Plasmodium vivax in Papua New Guinea reveals heterogeneous transmission dynamics and routes of migration amongst subdivided populations. Infect Genet Evol 2018, 58:83-95.

62. Shetty AC, Jacob CG, Huang F, Li Y, Agrawal S, Saunders DL, Lon C, Fukuda MM, Ringwald P, Ashley EA et al: Genomic structure and diversity of Plasmodium falciparum in Southeast Asia reveal recent parasite migration patterns. Nat Commun 2019, 10:2665. 
63. Jitthai N: Migration and malaria. Southeast Asian J Trop Med Public Health 2013, 44 Suppl 1:166200; discussion 306-167.

64. Abdullah NR, Barber BE, William T, Norahmad NA, Satsu UR, Muniandy PK, Ismail Z, Grigg MJ, Jelip J, Piera $\mathrm{K}$ et al: Plasmodium vivax population structure and transmission dynamics in Sabah Malaysia. PLoS One 2013, 8:e82553.

65. Koepfli C, Timinao L, Antao T, Barry AE, Siba P, Mueller I, Felger I: A Large Reservoir and Little Population Structure in the South Pacific. PLoS One 2013, 8:e66041.

66. Orjuela-Sanchez P, Sa JM, Brandi MC, Rodrigues PT, Bastos MS, Amaratunga C, Duong S, Fairhurst RM, Ferreira MU: Higher microsatellite diversity in Plasmodium vivax than in sympatric Plasmodium falciparum populations in Pursat, Western Cambodia. Exp Parasitol 2013, 134:318-326.

67. Getachew S, To S, Trimarsanto H, Thriemer K, Clark TG, Petros B, Aseffa A, Price RN, Auburn S: Variation in Complexity of Infection and Transmission Stability between Neighbouring Populations of Plasmodium vivax in Southern Ethiopia. PLoS One 2015, 10:e0140780.

68. Menegon M, Bardaji A, Martinez-Espinosa F, Botto-Menezes C, Ome-Kaius M, Mueller I, Betuela I, Arevalo-Herrera M, Kochar S, Kochar SK et al: Microsatellite Genotyping of Plasmodium vivax Isolates from Pregnant Women in Four Malaria Endemic Countries. PLoS One 2016, 11:e0152447.

69. Bahk YY, Kim J, Ahn SK, Na BK, Chai JY, Kim TS: Genetic Diversity of Plasmodium vivax Causing Epidemic Malaria in the Republic of Korea. Korean J Parasitol 2018, 56:545-552.

70. Socheat D, Denis MB, Fandeur T, Zhang Z, Yang H, Xu J, Zhou X, Phompida S, Phetsouvanh R, Lwin S et al: Mekong malaria. II. Update of malaria, multi-drug resistance and economic development in the Mekong region of Southeast Asia. Southeast Asian J Trop Med Public Health 2003, 34 Suppl 4:1102.

71. Zeng W, Bai Y, Wang M, Wang Z, Deng S, Ruan Y, Feng S, Yang Z, Cui L: Significant divergence in sensitivity to antimalarial drugs between neighboring Plasmodium falciparum populations along the eastern border of Myanmar. Antimicrob Agents Chemother 2017, 61:e01689-01616.

72. Fouet C, Kamdem C, Gamez S, White BJ: Genomic insights into adaptive divergence and speciation among malaria vectors of the Anopheles nili group. Evol Appl 2017, 10:897-906.

73. Joy DA, Gonzalez-Ceron L, Carlton JM, Gueye A, Fay M, McCutchan TF, Su XZ: Local adaptation and vector-mediated population structure in Plasmodium vivax malaria. Mol Biol Evol 2008, 25:12451252.

74. Sriwichai P, Samung Y, Sumruayphol S, Kiattibutr K, Kumpitak C, Payakkapol A, Kaewkungwal J, Yan G, Cui L, Sattabongkot J: Natural human Plasmodium infections in major Anopheles mosquitoes in western Thailand. Parasit Vectors 2016, 9:17.

75. Jongwutiwes S, Putaporntip C, Hughes AL: Bottleneck effects on vaccine-candidate antigen diversity of malaria parasites in Thailand. Vaccine 2010, 28:3112-3117.

76. Yuan L, Wang Y, Parker DM, Gupta B, Yang Z, Liu H, Fan Q, Cao Y, Xiao Y, Lee MC et al: Therapeutic responses of Plasmodium vivax malaria to chloroquine and primaquine treatment in northeastern Myanmar. Antimicrob Agents Chemother 2015, 59:1230-1235. 
77. Xu S, Zeng W, Ngassa Mbenda HG, Liu H, Chen X, Xiang Z, Li C, Zhang Y, Baird JK, Yang Z et al: Efficacy of directly-observed chloroquine-primaquine treatment for uncomplicated acute Plasmodium vivax malaria in northeast Myanmar: A prospective open-label efficacy trial. Travel Med Infect Dis 2019101499.

78. Kar NP, Kumar A, Singh OP, Carlton JM, Nanda N: A review of malaria transmission dynamics in forest ecosystems. Parasit Vectors 2014, 7:265.

79. Ferreira MU, Rodrigues PT: Tracking malaria parasites in the eradication era. Trends Parasitol 2014, 30:465-466.

80. Edwards HM, Sriwichai P, Kirabittir K, Prachumsri J, Chavez IF, Hii J: Transmission risk beyond the village: entomological and human factors contributing to residual malaria transmission in an area approaching malaria elimination on the Thailand-Myanmar border. Malar J 2019, 18:221.

\section{Tables}

Table 1. Genetic diversity of $P$. vivax populations at the China-Myanmar border (CMB) and western Thailand.

\begin{tabular}{|c|c|c|c|c|c|c|}
\hline Populations (N) & $\mathrm{Nh}$ & $\mathrm{H}_{\mathrm{E}} \pm \mathrm{SE}$ & $\mathrm{Na} \pm \mathrm{SE}$ & $\begin{array}{c}\text { Mean allelic } \\
\text { richness }\end{array}$ & $\begin{array}{c}\text { Multiclonal } \\
\text { infections (\%) }\end{array}$ & MOI \\
\hline CMB2004 (50) & 42 & $0.76 \pm 0.04$ & $10.80 \pm 1.20$ & 12.20 & 48.0 & 1.48 \\
\hline CMB2016 (52) & 34 & $0.66 \pm 0.08$ & $9.30 \pm 1.40$ & 8.80 & 30.7 & 1.33 \\
\hline $\begin{array}{l}\text { Thailand2012 } \\
\text { (50) }\end{array}$ & 39 & $0.80 \pm 0.03$ & $11.40 \pm 1.10$ & 11.20 & 40.0 & 1.50 \\
\hline $\begin{array}{l}\text { Thailand2015 } \\
\text { (54) }\end{array}$ & 47 & $0.86 \pm 0.02$ & $13.7 \pm 1.04$ & 12.80 & 23.7 & 1.36 \\
\hline
\end{tabular}

Nh, number of haplotypes; $\mathrm{H}_{\mathrm{E}}$, Expected heterozygosity; SE, standard error; Na, number of alleles.

\# Significant difference among the four populations $(\mathrm{P}<0.05$, Pearson Chi-square test)

Table 2. Effective population size $\left(N_{e}\right)$ of the P. vivax populations estimated using the SMM and IAM models.

\begin{tabular}{lllll}
\hline Populations & SMM & 95\%CI & IAM & 95\%CI \\
\hline CMB2004 & $\mathbf{1 2 8 6 2}$ & $5527-29299$ & $\mathbf{4 9 7 9}$ & $\mathbf{2 1 3 9 - 1 1 3 4 1}$ \\
CMB2016 & $\mathbf{6 0 1 4}$ & $2584-13700$ & $\mathbf{3 0 5 2}$ & $\mathbf{1 3 1 1 - 6 9 5 2}$ \\
Thailand2012 & $\mathbf{1 8 8 6 7}$ & $8108-42979$ & $\mathbf{6 2 8 9}$ & $\mathbf{2 7 0 2 - 1 4 3 2 6}$ \\
Thailand2015 & $\mathbf{4 3 7 2 4}$ & $18790-99601$ & $\mathbf{1 0 2 5 9}$ & $4408-23368$ \\
\hline
\end{tabular}

SMM, stepwise mutational model; IAM, infinite allele model.

95\% CI, 95\% confidence interval. 
Table 3. Multilocus linkage disequilibrium $\left(I^{S} A\right)$ in $\mathrm{P}$. vivax populations.

\begin{tabular}{|c|c|c|c|c|}
\hline \multirow[t]{2}{*}{ Population } & \multicolumn{2}{|c|}{ All 10 MS markers included } & \multicolumn{2}{|c|}{1 MS marker/chromosome ${ }^{\#}$} \\
\hline & All haplotypes & Unique haplotype & All haplotypes & Unique haplotype \\
\hline CMB2004 & $0.0320^{* * * *}$ & $0.0279 * * *$ & $0.0236^{*}$ & $0.0181 *$ \\
\hline CMB2016 & $0.0492^{* * * *}$ & $0.0492 * * *$ & $0.0329 * *$ & $0.0329 * *$ \\
\hline Thailand2012 & $0.0442^{* * * *}$ & $0.0442 * * *$ & $0.0366^{* * * *}$ & $0.0366 * * *$ \\
\hline Thailand2015 & $0.0679 * * *$ & $0.0199 * * *$ & $0.0586 * * *$ & $0.0261 * * *$ \\
\hline
\end{tabular}

$P_{A} A$, standardized index of association.

\# MS2 and MS12 were excluded from analysis.

$*, * *$, and $* * *$ indicates $P<0.05,0.01$, and 0.001 , respectively.

Table 4. Bottleneck analysis

\begin{tabular}{l|l|l|l|l}
\hline \multirow{2}{*}{ Populations } & \multicolumn{3}{|l|}{ SMM } & G-W statistic \\
\cline { 2 - 5 } & Excess-H $_{\mathrm{E}}$ & Deficient-H & 2-tails & Mean \pm SD \\
\hline CMB2004 & 0.991 & $0.01221^{*}$ & $0.0244^{*}$ & $0.14 \pm 0.13$ \\
\hline CMB2016 & 0.997 & $0.00342^{*}$ & $0.0068^{*}$ & $0.14 \pm 0.09$ \\
\hline Thailand2012 & 0.996 & $0.00488^{*}$ & $0.0097^{*}$ & $0.17 \pm 0.13$ \\
\hline Thailand2015 & 0.995 & $0.00684^{*}$ & $0.0137^{*}$ & $0.17 \pm 0.12$ \\
\hline
\end{tabular}

* For both Excess- $\mathrm{H}_{\mathrm{E}}$ and Deficient- $\mathrm{H}_{\mathrm{E}}, \mathrm{P}$-values were from one-tailed analysis and * indicates significance at $\mathrm{P}<$ 0.05 .

G-W, Garza-Williamson index; SD, standard deviation.

Table 5. Pairwise comparison of $\mathrm{F}_{\mathrm{ST}}$ among $P$. vivax populations from China-Myanmar border (CMB) and western Thailand

\begin{tabular}{lccc}
\hline Population & CMB2004 & CMB2016 & Thailand2012 \\
\hline CMB2016 & $0.081^{*}$ & & \\
Thailand2012 & $0.064^{*}$ & $0.169 *$ & \\
Thailand2015 & $0.172^{*}$ & $0.237^{*}$ & $0.133^{*}$ \\
\hline
\end{tabular}

*P values obtained after permutation test at $\mathrm{P}<0.01$.

\section{Figures}


A.
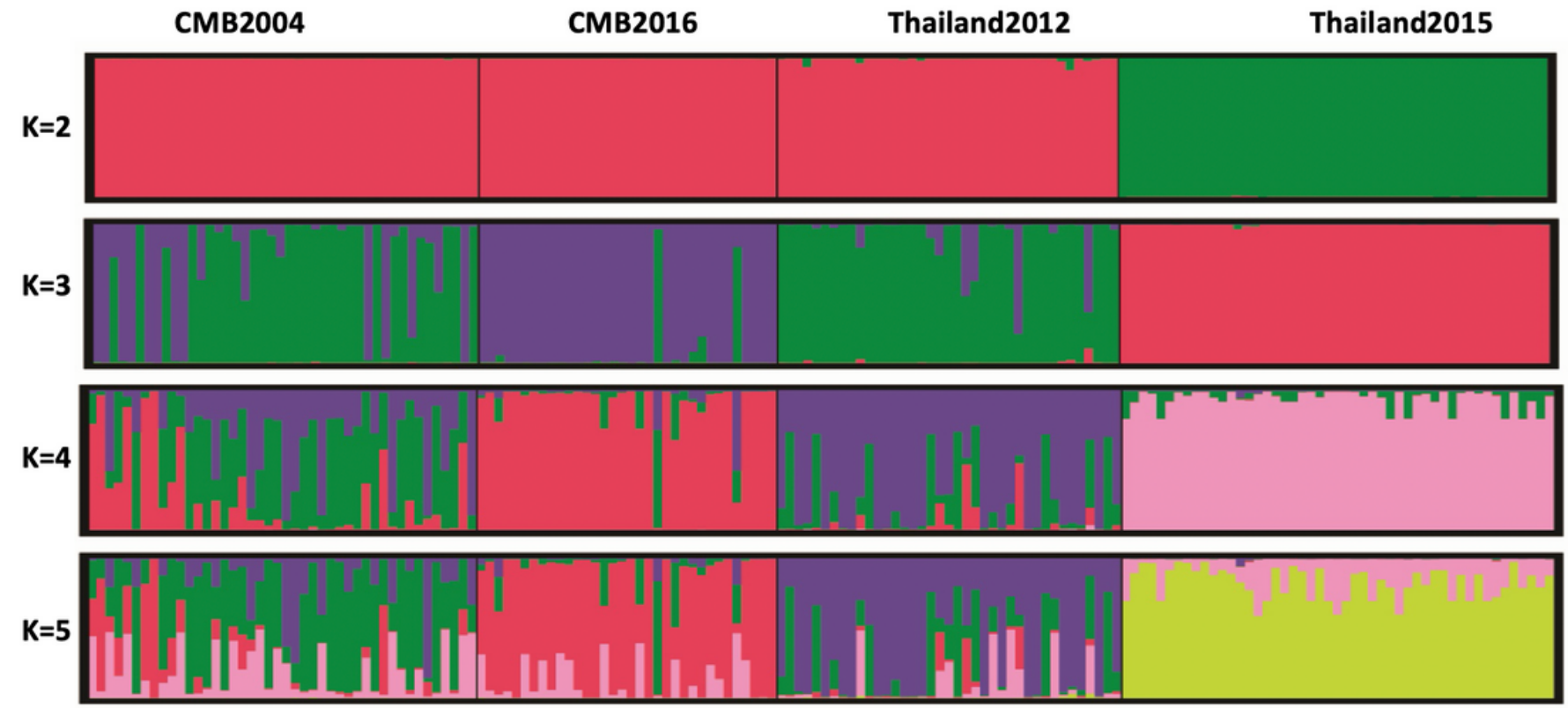

B.

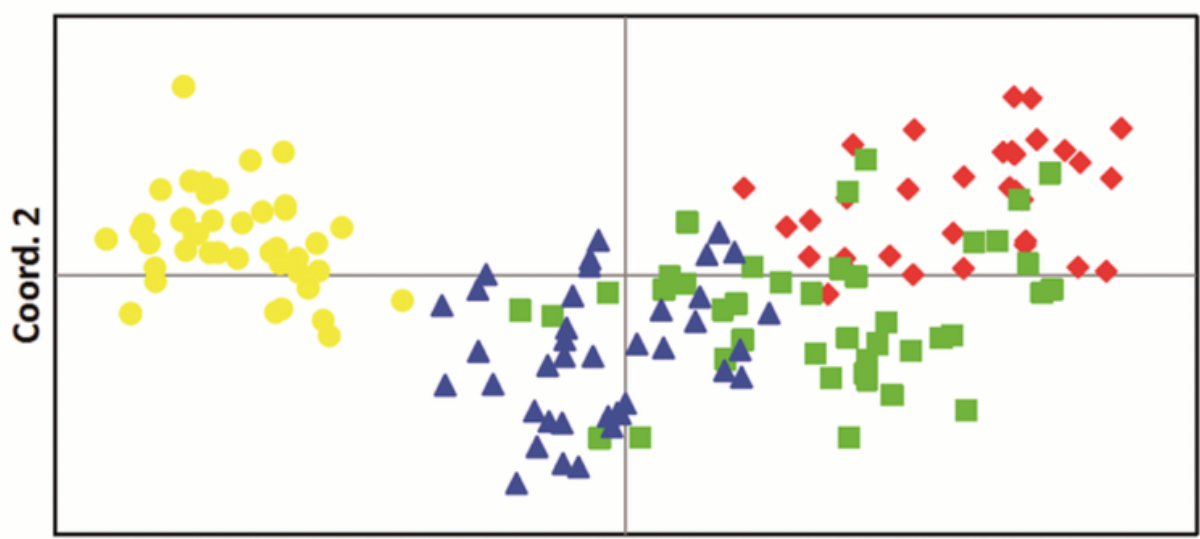

CMB2004

CMB2016

Thailand2012

Thailand2015

Coord. 1

Figure 1

A. Population genetic structure of P. vivax from four populations from the GMS $(K=2-5)$. The analysis assigns $P$. vivax haplotypes to a defined number of genetic clusters $(K)$ based on genetic distance. Vertical bars indicate individual P. vivax haplotype and colors represent the ancestry co-efficient (membership) within each cluster. B. Principal Coordinate Analysis of P. vivax haplotypes from four parasite populations. Colors indicate the geographic origin of each sample. CMB, China-Myanmar border. 

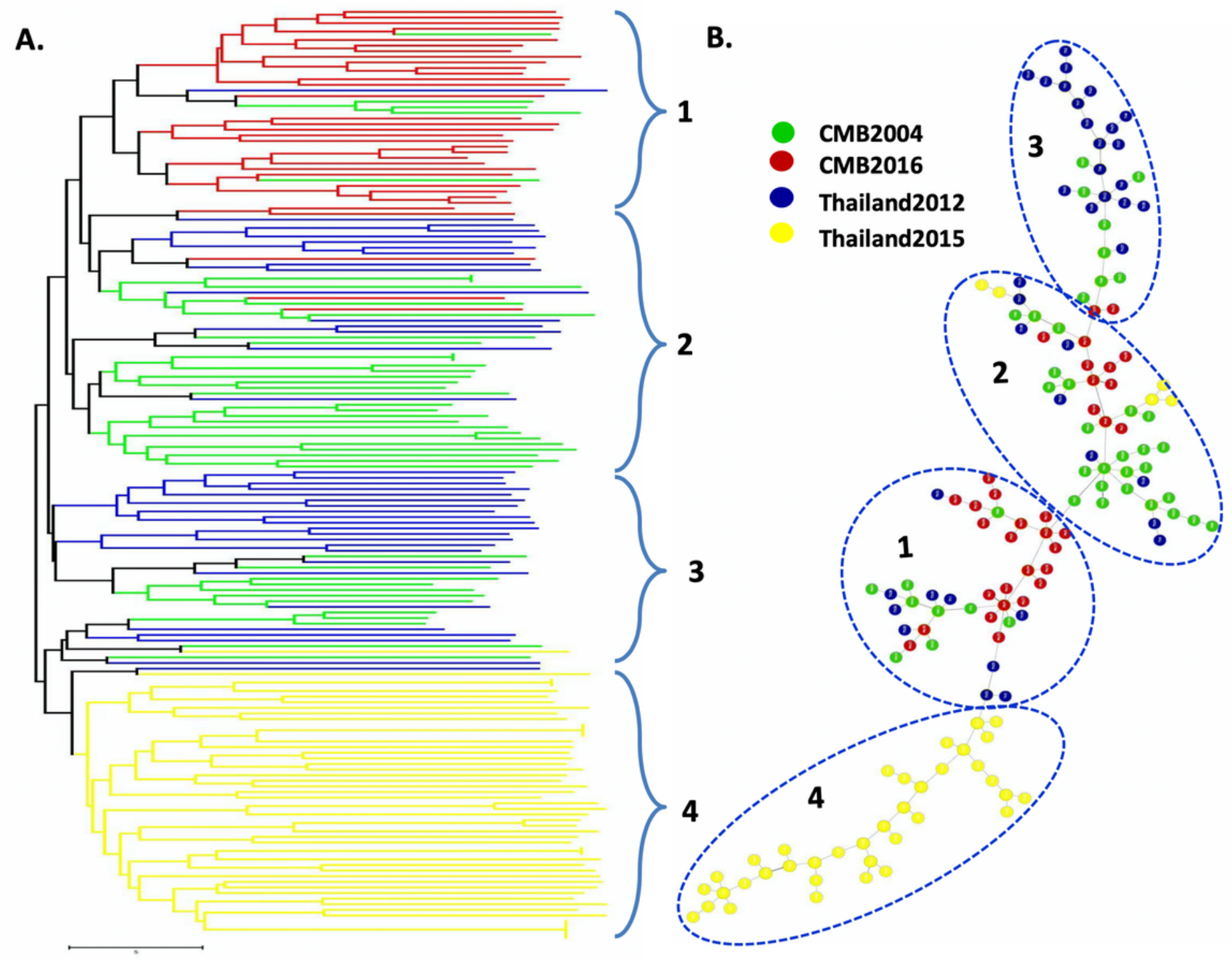

Figure 2

A. Phylogenetic analysis of P. vivax isolates from the four parasite populations. Relatedness amongst haplotypes was defined by calculating the pairwise distance and visualized by drawing an unrooted phylogenetic tree using a Neighbor-Joining method. B. Minimum spanning tree of parasite genotypes with each circle representing a haplotype. Colors indicate the geographic origin of each sample.

\section{Supplementary Files}

This is a list of supplementary files associated with this preprint. Click to download.

- S2Table.docx

- S1Table.docx

- S2Fig.pptx

- S1Fig.pptx 